\title{
Optical Humidity Sensor Based on Air Guided Photonic Crystal Fiber
}

\author{
M. Y. MOHD NOOR ${ }^{1,3^{*}}$, N. KHALILI ${ }^{2}$, I. SKINNER ${ }^{1}$, and G. D. PENG ${ }^{1}$ \\ ${ }^{1}$ School of Electrical Engineering \& Telecommunications, The University of New South Wales, Sydney, 2052, Australia \\ ${ }^{2}$ School of Civil \& Environmental Engineering, The University of New South Wales, Sydney, 2052, Australia \\ ${ }^{3}$ Faculty of Electrical Engineering, Universiti Teknologi Malaysia, Skudai, 81310, Johor, Malaysia \\ *Corresponding author: M. Y. MOHD NOOR E-mail: yusofnor@gmail.com
}

\begin{abstract}
We proposed a novel relative humidity (RH) sensor based on the air guided photonic crystal fiber (AGPCF) using the direct absorption spectroscopic method in this paper. The wavelength scanning around the water vapor absorption peak around $1368.59 \mathrm{~nm}$ was realized by injecting the saw-tooth modulated current to a distributed-feedback laser diode. A reference signal was used as a zero absorption baseline and to help reduce the interference from the distributed-feedback laser source and probed region. The humidity level was determined by the normalized voltage difference between the reference signal and sensor signal at the peak of water vapor absorption. We demonstrated that a length of 5-cm AGPCF with a fixed small air gap between the single mode fiber (SMF) and hollow core fiber as an opening achieved a humidity detection resolution of around $0.2 \% \mathrm{RH}$ over the range 0 to $90 \% \mathrm{RH}$ which did not require the use of any hygroscopic coating material.
\end{abstract}

Keywords: Air guided photonic crystal fiber, humidity sensor, wavelength scanning

\section{Introduction}

The measurement of the humidity is required in a range of areas, including meteorology, agriculture, clinical medicine, manufacturing, and civil engineering. Optical humidity fiber sensors offer specific advantages compared with their conventional counterparts such as small size and weight, immunity to electromagnetic interference, multi-sensors, and remote operation. A wide range of optical fiber humidity sensors have been reported in the literature review. Most of these fiber optic humidity sensors work on the basis of the hygroscopic sensing material coated over the optical fiber for modulation [1]. There are also fiber optic humidity sensors that do not use any hygroscopic material as shown by Lauer [2] with a direct spectroscopic technique in the open path by which the humidity sensing is based on the light attenuation at a certain wavelength by using the vertical-cavity surface-emitting laser (VCSEL) light source at the $1.84-\mu \mathrm{m}$ band. This humidity sensor is capable of measuring wide range of relative humidity variations but required the laser beam alignment and constraint of fiber components in that wavelength emission. Recently, the holey fiber has generated much interest in exploiting such fibers for sensing and spectroscopic analysis of gases [3-4]. A holey fiber based on the interferometer has been adapted for humidity sensing without the use of the hygroscopic coating too [5], but for the high humidity detection only. 
In this paper, we present a novel fiber optic humidity sensor based on the air guided photonic crystal fiber (AGPCF) with the direct absorption technique at $1369-\mathrm{nm}$ band to measure the relative humidity. A scanning saw-tooth distributed-feedback light source goes through the AGPCF. One end of the AGPCF is spliced to the standard single mode fiber (SMF). A fixed small gap on the other end of the AGPCF is used as an opening for a diffusion hole. A reference signal that acts as a zero absorption baseline is used to reduce the interference from the distributed-feedback laser source and probed region. The voltage difference between both signals at the peak of water vapor absorption changes to the variation of the humidity level. The distributed-feedback laser emitting at $1369 \mathrm{~nm}$ are highly suited for sensitive detection of the humidity cause by the strong absorption at this near infrared band and takes advantage of the mature telecommunication technology by which fiber coupled lasers and fiber components are readily available. To the best of our knowledge such the AGPCF based on spectroscopic technique at 1369-nm light emission to measure relative humidity which does not require any hygroscopic coating material is reported for the first time.

\section{Selection of water vapor line absorption}

A critical important step in the design of an optical humidity sensor based on the direct absorption technique is the selection of line absorption. Proper absorption line selection can significantly improve the accuracy and performance of the sensor. There are number of water vapor absorption bands that have been considered for study, all of which are listed in the HITRAN database [6]. There are many bands of water vapor transitions over the range of wavelengths from $1000 \mathrm{~nm}$ and $8000 \mathrm{~nm}$ but in our work, the focus of wavelength operation is below $2000 \mathrm{~nm}$, as shown in
Fig. 1. These water vapor spectra are extracted from the latest update of the HITRAN database for water vapor which is the HITRAN 2008 molecular spectroscopic database. The wavelength that have the higher line strength $(S)$ represents relatively stronger absorption feature at that specific wavelength. There are 3 major absorption bands appeared from $1000 \mathrm{~nm}$ to $2000 \mathrm{~nm}$. The strongest absorption band of water vapor in the near infrared region is around $1800 \mathrm{~nm}$. Although the absorption band in the $1800 \mathrm{~nm}$ are stronger than the other combination and overtone vibrational transitions in the near infrared region below $2000 \mathrm{~nm}$, the laser and fiber technologies are less mature in the this wavelength band. Accordingly, we limit the choice of transitions to the $1000-\mathrm{nm}$ to $1600 \mathrm{~nm}$ region where laser and fiber optics technologies are well developed and readily available.

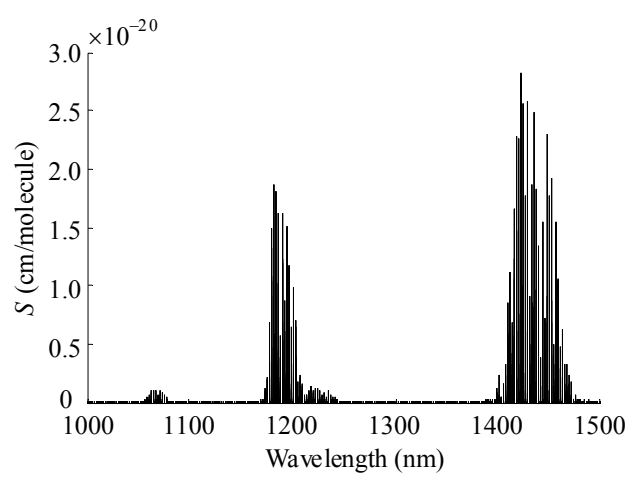

Fig. 1 Water vapor absorption spectra in the $1000-\mathrm{nm}$ to 2000-nm region based on the HITRAN database.

Specifically, we have chosen to probe the 1368.59-nm water vapor absorption feature to measure the relative humidity which arises from the $2_{12} \leftarrow 3_{13}$ rotational line within the $\mathrm{v}_{3}+\mathrm{v}_{2}$ vibrational band. This is because the reported line strength value for the 1368.59-nm absorption feature in the HITRAN database is $1.8 \times 10^{-20} \mathrm{~cm} /$ molecule as shown in Fig. 2 which is one of the highest in the region of $1000 \mathrm{~nm}$ to $16000 \mathrm{~nm}$ and includes only one transition around this band to avoid the transition overlap with the neighboring transitions when the pressure is broadened [7] that could cause 
complicated absorption measurement. First time that the absorption line of water vapor around 1369-nm band is used to measure the relative humidity.

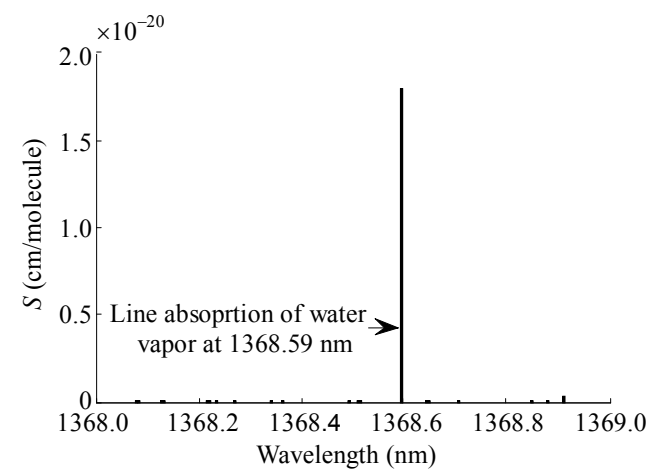

Fig. 2 Chosen water vapor line absorption at $1368.59 \mathrm{~nm}$.

\section{Principle}

A common way to relate the amount of water vapor present in the environment is to take the ratio of the actual water vapor pressure to the saturation water vapor pressure at a specific temperature. The resultant term, known as the relative humidity $(\mathrm{RH})$, simply represents the ratio of the amount of water vapor present in the atmosphere to the maximum amount the atmosphere can hold and if often expressed as a percentage using the following equation:

$$
R H=\frac{P_{v}}{P_{w S}} \times 100 \%
$$

where $P_{v}$ is the partial pressure of water vapor, and $P_{w s}$ is the saturation water vapor pressure.

In this optical absorption humidity sensor, the

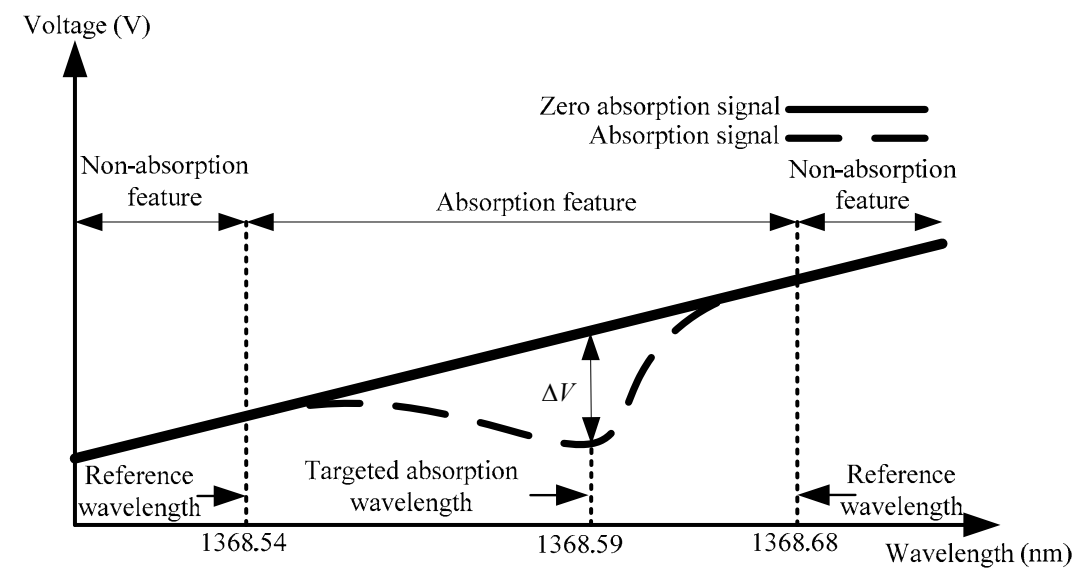

Fig. 3 Illustration of distributed-feedback laser saw-tooth modulation in one scanning period. presence of water vapor is determined according to the Beer-Lambert law by which the amount of water vapor is related to the power output [8] as

$$
P=P_{o} e^{-\alpha C L}
$$

where $P$ is the power of the output signal, $P_{o}$ is the power of the received signal, $\alpha$ is the absorption coefficient of water vapor, $C$ is the amount of water vapor, and $L$ is the absorption path length. The power output will decrease when the relative humidity increases and vice versa with the fixed temperature condition. The holey fiber is used as a sensing element. The transmission light that goes through the holey fiber will be attenuated or absorbed at certain wavelength caused by water vapor inside the holey fiber. A small gap between the SMF and holey fiber is exposed to the atmosphere to allow gas in and out of the holey fiber. By using a wide range of scanning the wavelength scheme, a narrow-linewidth of the distributed-feedback laser is tuned not only the entire absorption feature but also the non-absorption feature of water vapor as shown in Fig. 3 using saw-tooth modulation. A reference signal is constructed by using a reference point of non-absorption of water vapor at both sides of the absorption feature in one scanning period. The voltage change at the center of the absorption peak of water vapor varies when the humidity level changes and is defined as

$$
\begin{aligned}
\Delta V & =\text { the power of the reference signal } \\
& \text {-the power of the sensor signal }
\end{aligned}
$$


The advantage of using this reference signal as the zero absorption baseline is that the resulting $\Delta V$ signal is immune to the outside affection such as noise of the laser source, fiber loss, and unwanted attenuation in the probed region. This is because all interference will affect both the sensor and reference signals, and therefore the $\Delta V$ signal intensity indicates the amount of water vapor independently of the received laser power.

\section{Experiment}

Figure 4 shows the setup to detect the relative humidity in the atmospheric pressure and room temperature. The water vapor absorption band centered around $1368 \mathrm{~nm}$ is one of the strongest in the near infrared region based on the HITRAN database, and therefore, a wavelength scan of the water vapor absorption peak around $1368.59 \mathrm{~nm}$ is chosen and realized by the saw-tooth modulation current at $200 \mathrm{~Hz}$ which is injected into the distributed-feedback laser and the output light from the hollow core fiber detected at the photo-detector [Optiphase V500, the signal generation and acquisition, as well as the signal processing is done using a PC equipped with data acquisition (DAQ) card (model: NI-6215)].

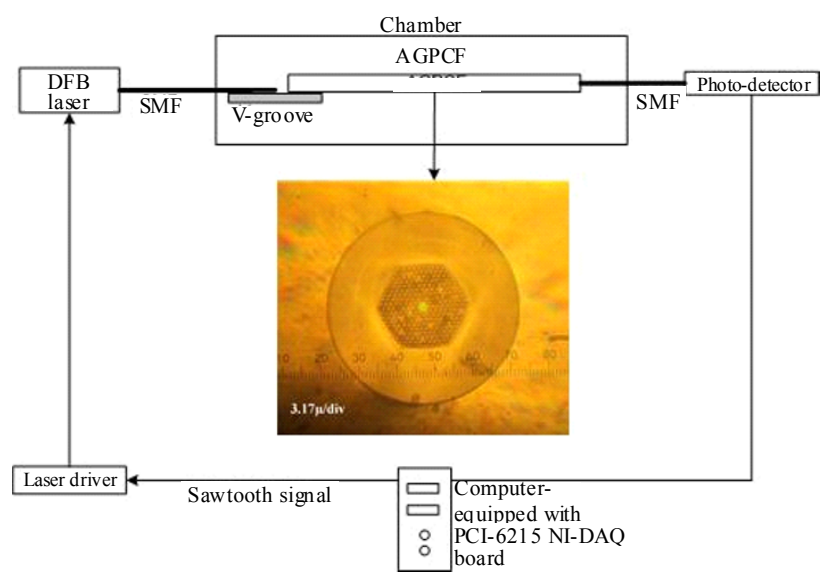

Fig. 4 System design and AGPCF cross section.

The scanning range of the absorption feature is around $0.25 \mathrm{~nm}$. The $\Delta V$ signal intensity is used to gain a meaningful humidity measurement. In this work, the AGPCF is picked as the holey fiber as this
AGPCF can guide over the $98 \%$ of the light in the holey regions of the fiber [9]. The detection of the humidity can be realized in better sensitivity even at the low humidity region as almost all the transmission light of the AGPCF overlaps with the air. The AGPCF consists of 8 rings of air holes arranged in a hexagonal pattern and one hollow core as depicted in Fig. 4 which guides light by a photonic bandgap effect. The diameter of the cladding is around $50 \mu \mathrm{m}$. One end of the AGPCF is spliced to a standard optical fiber (SMF-28) using a fusion splicing machine with a splice loss around $1 \mathrm{~dB}$. A default program for splicing single mode fibers with optimized parameters is used to ensure repeatability of the process. On the other end, a small gap is adjusted to about $50 \mu \mathrm{m}$ [10] for efficient filling while keeping good signal coupling efficiency using 3D stages and fixed to the $\mathrm{V}$-groove for a permanent gap between the fibers. This gap is open to ambient atmospheres that act as diffusion holes. The absorption path is equal to the length of the AGPCF that is $5 \mathrm{~cm}$. The humidity response of the sensor is studied at the room temperature around $25{ }^{\circ} \mathrm{C}$ and normal atmospheric pressure by placing it in the chamber. The humidity in the chamber varies by using the wet air (generated by the humidifier) and dry air (dry nitrogen gas). An electronic relative humidity sensor is used to monitor the temperature and humidity internally for calibration.

\section{Results}

The scanning sensor signal is averaging for 10 times to reduce the noise and smooth the signals as shown in Fig. 5. The dip of the absorption at $1368.59 \mathrm{~nm}$ can be seen clearly in one scanning period. The absorption in the sensor signal in this figure is caused by the room humidity, and a reference signal is constructed based on both wings of absorption feature where there is no absorption of water vapor in these points of the region by using combinations of the waveform measurement and 
generation function in the LabVIEW. By analyzing the voltage difference between the sensor signal and reference signal at peak absorption of water vapor $(\Delta V)$ around 1368.59-nm wavelength using a LabVIEW peak-detector function, a meaningful relative humidity reading can be recorded in a normalized value. In this way, the real time measurement can be achieved without further data processing. In Fig. 6, the sensor is calibrated with the electronic sensor from 0 to $90 \% \mathrm{RH}$. It is found that this sensor has a detection range from around 0 to $90 \%$ RH with the linear response. In order to estimate the minimum detection of the humidity level, two measurements of the fixed humidity at $90 \% \mathrm{RH}$ and $5 \% \mathrm{RH}$, respectively are performed for 350 s inside the chamber as shown in Fig. 7, and the statistical fluctuation over time is calculated. The mean voltage $(\mu)$ and standard deviation $(\sigma)$ were found to be 0.123 and 0.000276 . The signal to noise ratio (SNR) was then calculated to be 447 by dividing between the $\mu$ value and $\sigma$ value to estimate the sensitivity of the sensor [11]. The sensitivity in term of the minimum detection of the relative humidity for an SNR of unity is then estimated to be $0.2 \% \mathrm{RH}$. To test the reversibility characteristic of the sensor, several cycles between two different relative humidity levels have been tested. These relative humidity levels change alternatively inside the chamber and switched back and forth for several cycles. During such a process, the sensor shows a good reversibility as shown in Fig. 8.

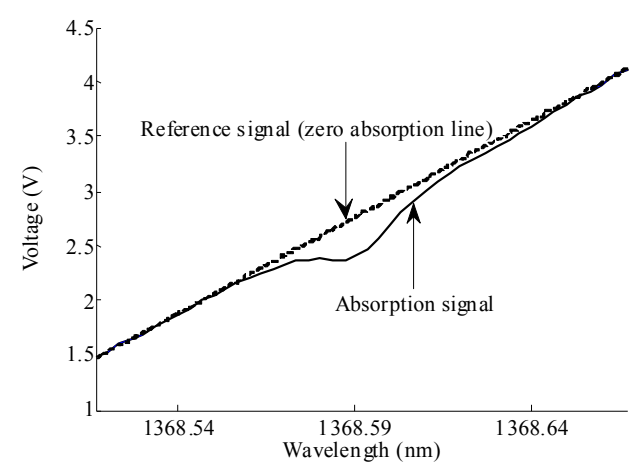

Fig. 5 Absorption and reference signal.

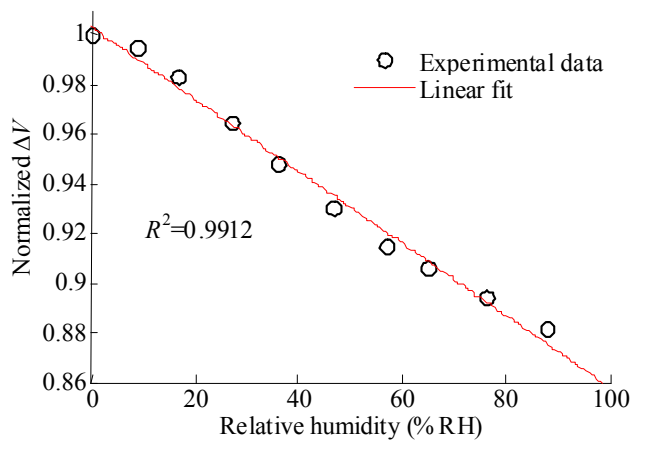

Fig. 6 Range detection of the sensor.

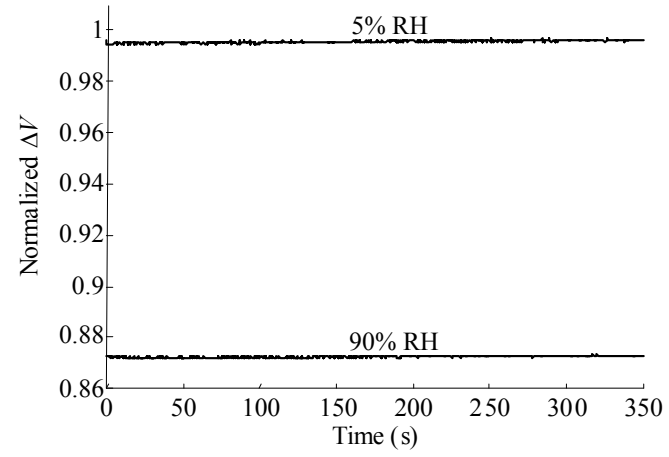

Fig. 7 Normalized $\Delta V$ signal for a fixed humidity level at $90 \% \mathrm{RH}$ and $5 \% \mathrm{RH}$.

Practically, the wavelength scanning modulation at $200 \mathrm{~Hz}$ applied to the distributed-feedback laser would help to reduce interferometric noises caused by multiple reflection, but there appears a small fringe that can shift when the fiber bends and could not be eliminated. Hence, external disturbances such as the fiber bend would cause shift in the fringes and induce error in measurement. However, the results presented here were obtained in the controlled laboratory environment to minimize the environmental disturbance.

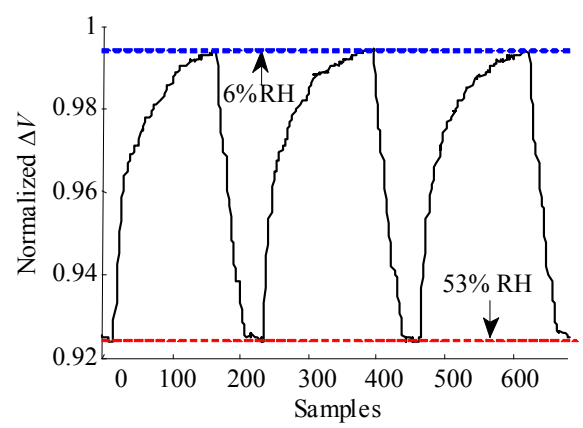

Fig. 8 Reversibility of the sensor between $53 \% \mathrm{RH}$ and $6 \% \mathrm{RH}$ at the room temperature. 


\section{Conclusions}

We have demonstrated a novel relative humidity optical fiber sensor based on the direct absorption spectroscopic technique at $1369-\mathrm{nm}$ band using the AGPCF and therefore does not require the use of any hygroscopic coating material. A saw-tooth scanning wavelength scheme is applied for humidity sensing, and the relative humidity level is based on the normalized value of voltage difference between the reference signal and sensor signal at the peak of the water vapor absorption. A 5-cm AGPCF with a small gap as a diffusion hole demonstrated a high humidity resolution or sensitivity of around $0.2 \% \mathrm{RH}$ in the humidity measurement condition from 0 to $90 \% \mathrm{RH}$.

Open Access This article is distributed under the terms of the Creative Commons Attribution License which permits any use, distribution, and reproduction in any medium, provided the original author(s) and source are credited.

\section{References}

[1] T. L. Yeo, T. Sun, and K. T. V. Gratan, "Fiber-optic sensor technologies for humidity and moisture measurement," Sensors and Actuators A: Physical, vol. 144, no. 2, pp. 280-295, 2008.

[2] C. Lauer, S. Szalay, G. Bohm, C. Lin, F. Kohler, and M. C. Amann, "Laser hygrometer using a vertical vertical-cavity surface-emitting laser (VCSEL) with an emission wavelength of $1.84 \mu \mathrm{m}$," IEEE
Transactions on Instrumentation and Measurement, vol. 54, no. 3, pp. 1214-1218, 2005.

[3] W. Jin, H. F. Xuan, and H. L. Ho, "Sensing with hollow-core photonic bandgap fibers," Measurement Science and Technology, vol. 21, no. 9, pp. 4014-4026, 2010.

[4] Y. L. Hoo, S. Liu, H. L. Ho, and W. Jin, "Fast response microstructured optical fiber methane sensor with multiple side-openings," IEEE Photonic Technology Letters, vol. 22, no. 5, pp. 296-298, 2010.

[5] J. Mathew, Y. Semenova, G. Rajan, and G. Farrell, "Humidity sensor based on photonic crystal fiber," Electronic Letters, vol. 46, no. 19, pp. 1341-1343, 2010.

[6] L. S. Rothman, I. E. Gordon, A. Barbe, D. C. Benner, P. F. Bernath, M. Birk, et al., "The HITRAN database 2008 molecular spectroscopic database," Journal of Quatitative Spectroscopy and Radiative Transfer, vol. 110, no. 2, pp. 533-572, 2009.

[7] A. Ray, A. Bandyopadhyay, B. Ray, D. Biswas, and P. N. Ghosh, "Line-shape study of water vapor by tunable diode laser spectrometer in the 822-832 nm wavelength region," Applied Physics B: Lasers and Optics, vol. 79, no. 7, pp. 915-921, 2004.

[8] H. Wang. Q. Wang, J. Chang, X. Zhang, S. Zhang, and $\mathrm{J}$. Ni, "Measurement technique for methane concentration by wavelength scanning of a distributed-feedback laser," Laser Physics, vol. 18, no. 4, pp. 491-494, 2008

[9] A. M. Cubillas, J. M. Lazaro, M. Silvo-Lopez, O. M. Conde, M. Petrovich, and J. M. Lopez-Higuera, "High sensitive methane sensor based on a photonic bandgap fiber," presented at Third European Workshop on Optical Fiber Sensors (EWOFS 2007), Napoli, Italy, 2007.

[10] T. Ritari, J. Tuominen, and H. Ludvigsen, "Gas sensing using air-guiding photonic bandgap fibers," Optics Express, vol. 12, no. 17, pp. 4080-4087, 2004.

[11] W. Demtroder, Laser Spectroscopy: Basic Concepts and Instrumentation. New York: Springer, 1993, pp. 369-372. 